\title{
SISTEMA DE EVALUACIÓN POR COMPETENCIAS EN ESTUDIANTES DE LA ESCUELA PROFESIONAL DE MATEMÁTICA DE LA UNIVERSIDAD NACIONAL JORGE BASADRE GROHMANN
}

\author{
SYSTEM OF EVALUATION FOR COMPETENCES IN STUDENTS OF THE \\ PROFESIONAL SCHOOL OF MATHEMATICS OF THE NATIONAL UNIVERSITY \\ JORGE BASADRE GROHMANN
}

\author{
Humberto Vargas Pichon ${ }^{14}$, \\ Luis Solorzano Espinola ${ }^{15}$, \\ Ita Huaman Guzman ${ }^{16}$
}

\section{RESUMEN}

Tipo de estudio: cuasiexperimental. Objetivo general: Determinar en qué medida un Sistema de evaluación por competencias orientado hacia una Educación de calidad influye significativamente en el rendimiento académico en estudiantes de la Escuela Profesional de Matemática de la Universidad Nacional Jorge Basadre Grohmann de Tacna - 2017. Población y muestra: La población comprendió 78 estudiantes y la muestra conformada por 39 estudiantes cuya selección fue usando un muestreo aleatorio estratificado con afijación proporcional. Resultado: Existe una diferencia significativa entre la media de calificaciones en rendimiento académico de alumnos del Grupo experimental, sin aplicar y después de aplicar un Sistema de evaluación basado en competencias orientado hacia una Educación de calidad en estudiantes de la Escuela Profesional de Matemática de la Universidad Nacional Jorge Basadre Grohmann de Tacna - 2017 ( $p$-Valor $=0,000<0,05$ para el II semestre, $p$ Valor $=0,018<0,05$ para el III semestre, $p$-Valor $=0,042<0,05$ para el V semestre y $p$-Valor $=0,000$ $<0,05$ para el VI semestre).

Palabras Clave: Sistema de evaluación, rendimiento académico y competencia.

\section{ABSTRACT}

Type of study: quasi-experimental. Course objective: Determine the extent to which a system of evaluation for competences oriented towards quality education significantly influences the academic performance of students of the Professional School of Mathematics of the National University Jorge Basadre Grohmann de Tacna - 2017. Population and sample: The population comprised 78 students and the sample consisted of 39 students whose selection was using a stratified random sampling with proportional allocation. Result: There is a significant difference between the average of grades in academic performance of students of the experimental group, without applying and after applying a system of evaluation based on competencies oriented towards a quality education in students of the Professional School of Mathematics of the University National Jorge Basadre Grohmann de Tacna 2017 ( $p$-Value $=0,000<0,05$ for the II semester, $p$-Value $=0,018<0,05$ for the III semester, $p$-Value $=$ $0,042<0,05$ for the $V$ semester and $p$-Value $=0,000<0,05$ for the sixth semester).

Keywords: System evaluation, academic performance and competence.

\footnotetext{
14 Doctor en Administración de la Educación

Docente de la Facultad de Ciencias - Universidad Nacional Jorge Basadre Grohmann

${ }^{15}$ Maestro en Ciencias con mención en Tecnología Educativa

${ }^{16}$ Licenciada en Obstetricia MINSA - Tacna
} 


\section{IINTRODUCCIÓN}

En la actualidad las instituciones de educación superior se encuentran inmersas en procesos profundos de análisis y reflexión debido a las exigencias que enfrentan por los cambios significativos en su entorno. A diferencia de épocas anteriores, el conocimiento en sus distintos saberes se ha transformado en un factor decisivo para la inserción a un mundo globalizado y a un mercado internacional, en donde el intercambio de capital humano, de bienes materiales y bienes culturales, se ha convertido en una necesidad primordial de la sociedad actual. Frente a esta revolución económica, política y sociocultural, las instituciones de educación superior se plantean procesos de grandes transformaciones en sus sistemas académicos, particularmente en sus estructuras curriculares que requieren de un alto grado de articulación y concreción para poder procesar los constantes cambios.

\section{El espacio europeo de educación superior}

Con las Declaraciones de Sorbona (1998), y Bolonia (1999), se marcó el inicio del proceso de concertación para la transformación de la educación superior más trascendental del siglo XX en la mayoría de los países del continente europeo. Esta propuesta más conocida como "el Proceso de Bolonia" o "Convergencia Europea", procuraba para el año 2010, haber conformado el Espacio Europeo de Educación Superior (EEES). Así, se desarrolló el Proyecto Tuning Educational Structures in Europe (PTESE); una iniciativa de las universidades con el fin de encontrar conjuntamente puntos comunes de referencia que facilitaran la libre circulación internacional de los estudiantes y profesores en estos centros de educación superior tal como lo declara el Proceso de Convergencia (Lorenzana, 2012, p. 12-13).

Para el desarrollo del Proyecto Tuning, se tomó como marco de referencia las experiencias acumuladas de los programas ERASMUS-SOCRATES, especialmente en lo que respecta al Sistema Europeo de Transferencia y Acumulación de Créditos
(ECTS). Para las instituciones de educación superior, el PTESE, fue un punto de partida para la homologación de las estructuras curriculares y contenidos de estudio que la colectividad académica donde más de 100 expertos europeos, definieron en ese espacio de diálogo y reflexión, los posibles perfiles educativos y profesionales que la sociedad exige y el nivel de formación que los estudiantes en términos de competencias y resultados de aprendizaje deberían alcanzar (Lorenzana, 2012, p. 17). EI PTESE, definió el término Competencia como "Una combinación dinámica de atributos, en relación a procedimientos, habilidades, actitudes y responsabilidades, que describen los encargados del aprendizaje de un programa educativo o lo que los alumnos son capaces de demostrar al final de un proceso educativo" (Bravo, 2007, p. 13). En tal sentido, los expertos académicos organizaron las competencias en un conjunto de conocimientos, habilidades, destrezas y actitudes, las cuales se espera que los estudiantes adquieran y dominen durante, o al término de un ciclo de estudio; proceso corto o largo de aprendizaje; igualmente, identificadas con programas completos de estudio o con unidades individuales de aprendizaje (Lorenzana, 2012, p. 18).

\section{Contexto latinoamericano}

Pese a las marcadas diferencias que le caracterizan, América Latina, no podría mantenerse al margen de los vertiginosos cambios y negarse la oportunidad de encontrar un lugar más equitativo y justo, tanto en la economía regional como en el mercado mundial. La transformación de la educación superior en Latinoamérica estaba sujeta a una serie de factores o demandas que deben tomarse en cuenta al momento de impulsar las reformas si se espera obtener cambios genuinos, significativos y perecederos. Ello es, que promuevan el desarrollo socio económico de los países, y que ofrezcan oportunidades en la mejora de la calidad de vida de sus ciudadanos. Así, la UNESCO, a partir de la década de los "90s" llevó a cabo un proceso de consulta con 


\section{IINTRODUCCIÓN}

En la actualidad las instituciones de educación superior se encuentran inmersas en procesos profundos de análisis y reflexión debido a las exigencias que enfrentan por los cambios significativos en su entorno. A diferencia de épocas anteriores, el conocimiento en sus distintos saberes se ha transformado en un factor decisivo para la inserción a un mundo globalizado y a un mercado internacional, en donde el intercambio de capital humano, de bienes materiales y bienes culturales, se ha convertido en una necesidad primordial de la sociedad actual. Frente a esta revolución económica, política y sociocultural, las instituciones de educación superior se plantean procesos de grandes transformaciones en sus sistemas académicos, particularmente en sus estructuras curriculares que requieren de un alto grado de articulación y concreción para poder procesar los constantes cambios.

\section{El espacio europeo de educación superior}

Con las Declaraciones de Sorbona (1998), y Bolonia (1999), se marcó el inicio del proceso de concertación para la transformación de la educación superior más trascendental del siglo XX en la mayoría de los países del continente europeo. Esta propuesta más conocida como "el Proceso de Bolonia" o "Convergencia Europea", procuraba para el año 2010, haber conformado el Espacio Europeo de Educación Superior (EEES). Así, se desarrolló el Proyecto Tuning Educational Structures in Europe (PTESE); una iniciativa de las universidades con el fin de encontrar conjuntamente puntos comunes de referencia que facilitaran la libre circulación internacional de los estudiantes y profesores en estos centros de educación superior tal como lo declara el Proceso de Convergencia (Lorenzana, 2012, p. 12-13).

Para el desarrollo del Proyecto Tuning, se tomó como marco de referencia las experiencias acumuladas de los programas ERASMUS-SOCRATES, especialmente en lo que respecta al Sistema Europeo de
Transferencia y Acumulación de Créditos (ECTS). Para las instituciones de educación superior, el PTESE, fue un punto de partida para la homologación de las estructuras curriculares y contenidos de estudio que la colectividad académica donde más de 100 expertos europeos, definieron en ese espacio de diálogo y reflexión, los posibles perfiles educativos y profesionales que la sociedad exige y el nivel de formación que los estudiantes en términos de competencias y resultados de aprendizaje deberían alcanzar (Lorenzana, 2012, p. 17). EI PTESE, definió el término Competencia como "Una combinación dinámica de atributos, en relación a procedimientos, habilidades, actitudes y responsabilidades, que describen los encargados del aprendizaje de un programa educativo o lo que los alumnos son capaces de demostrar al final de un proceso educativo" (Bravo, 2007, p. 13). En tal sentido, los expertos académicos organizaron las competencias en un conjunto de conocimientos, habilidades, destrezas y actitudes, las cuales se espera que los estudiantes adquieran y dominen durante, o al término de un ciclo de estudio; proceso corto o largo de aprendizaje; igualmente, identificadas con programas completos de estudio o con unidades individuales de aprendizaje (Lorenzana, 2012, p. 18).

\section{Contexto latinoamericano}

Pese a las marcadas diferencias que le caracterizan, América Latina, no podría mantenerse al margen de los vertiginosos cambios y negarse la oportunidad de encontrar un lugar más equitativo y justo, tanto en la economía regional como en el mercado mundial. La transformación de la educación superior en Latinoamérica estaba sujeta a una serie de factores o demandas que deben tomarse en cuenta al momento de impulsar las reformas si se espera obtener cambios genuinos, significativos y perecederos. Ello es, que promuevan el desarrollo socio económico de los países, y que ofrezcan oportunidades en la mejora de la calidad de vida de sus ciudadanos. Así, la 
UNESCO, a partir de la década de los "90s" llevó a cabo un proceso de consulta con diferentes actores de la sociedad tanto a nivel mundial como a nivel regional, que le permita elaborar las políticas educativas de la educación superior. Tal fue el caso de la reunión internacional de reflexión "Los nuevos roles de la educación superior en América Latina" sostenida en Caracas, Venezuela en mayo de 1991, y organizada por el Centro Regional para la Educación en América Latina y el Caribe (CRESALC) (Lorenzana, 2012, p. 19-23).

Consecuentemente, se llevó a cabo la Conferencia Regional sobre Políticas y Estrategias para la Transformación de la Educación Superior en América Latina en la República de Cuba en noviembre de 1996 con 26 países participantes. En este espacio de reflexión, se presentaron propuestas concretas respecto a los principios fundamentales que, a juicio de los participantes, eran los necesarios para impulsar la reforma. Igualmente, se elaboró un plan de acción que condujera progresivamente, al desarrollo de la educación permanente y sin fronteras, en un nuevo contexto de apoyo y cooperación nacional e internacional para tal efecto (Lorenzana, 2012, p. 23-).

En el marco de reflexión sobre la necesidad de transformación de la educación superior, y en el espacio de colaboración de la Comisión Europea a través del programa ALFA, surgío el proyecto Tuning-América Latina; "trabajo conjunto que buscó y construyó lenguajes y mecanismos para la comprensión recíproca de los sistemas de enseñanza superior, que facilitaron los procesos de reconocimiento de carácter transnacional y regional". El proyecto Tuning-América Latina se desarrolló con la participación de más de 200 académicos de 19 países de la región, con el propósito de rescatar el papel de la educación superior como centro del pensamiento, del debate, la cultura y de la innovación. Uno de los aportes más significativos del proyecto fue la introducción de la metodología para la comprensión y comparación del currículo introduciendo los conceptos: resultados de aprendizaje y competencias. El primer concepto se define como "el conjunto de competencias que incluye conocimientos, comprensión y habilidades y que se espera que el estudiante domine, comprenda y demuestre después de completar un proceso corto o largo de aprendizaje" (Bravo, 2007 p. 3). Las competencias por otra parte, han sido categorizadas en dos grandes grupos: competencias genéricas, que en principio son independientes del área de estudio y competencias específicas; propias de cada disciplina del conocimiento (Lorenzana, 2012, p. 24-25).

\section{Contexto peruano}

Podemos hacer mención al "I Encuentro Internacional Universitario EL CURRICULO POR COMPETENCIAS EN LA EDUCACION SUPERIOR: Ponencias y debate", organizado los días 23 y 24 de octubre del 2014 y llevado a cabo en la Pontificia Universidad Católica del Perú (Carrillo, 2015, p. 145-147). En dicho evento uno de los temas tratados fue: La evaluación por competencias. Se llegó a las siguientes conclusiones:

1. Acerca de la evaluación a nivel de sistema, o macrocurricular: Autoevaluación para la acreditación. Condiciones para la implementación: Capacitación docente para que todos comprendan la lógica y proceso del cambio, y Evaluación del currículo.

2. Aspectos que se debe tener en cuenta en el desempeño del docente universitario:

- Competencias del perfil del docente en la educación superior (Temuco): Planificación de la enseñanza (probidad). - Estrategias de enseñanza (tríada tecnología aplicada a la disciplina carrera, asistente y profesor). - Cultura de evaluación. - Comunicación. - Reflexión sobre la propia práctica. - Uso de TIC.

- Evaluación docente: - Tipo 360 (autoevaluación, evaluación del jefe del departamento y del decano, evaluación de colegas y evaluación de estudiantes). Módulos de capacitación o desarrollo docente.

- Taxonomía de Guskey (2002): Satisfacción o reacciones del docente. - 
Tabla 1. Estadísticos descriptivos de la Asignatura Estadística Descriptiva

\begin{tabular}{|l|c|c|c|c|c|}
\hline & $\mathrm{N}$ & Mínimo & Máximo & Media & Desv. típ. \\
\hline PROMEDIO FINAL & 14 & 2,0 & 13,0 & 8,571 & 3,3447 \\
ESTADÍSTICA & 14 & & & & \\
$\begin{array}{l}\text { DESCRIPTIVA } \\
\text { N válido (según lista) }\end{array}$ & 14 & & & & \\
\hline
\end{tabular}

Fuente: Registro Académico de la $\mathrm{FACl}$, diciembre 2017

Tabla 2. Estadísticos descriptivos de la Asignatura Computación Matemática I

\begin{tabular}{|l|c|c|c|c|c|}
\hline & $\mathrm{N}$ & Mínimo & Máximo & Media & Desv. típ. \\
\hline PROMEDIO & 14 & 10,00 & 15,00 & 12,4286 & 1,55486 \\
$\begin{array}{l}\text { FINAL } \\
\text { COMPUTACIÓN } \\
\text { MATEMÁTICA I } \\
\begin{array}{l}\text { N válido (según } \\
\text { lista) }\end{array}\end{array}$ & 14 & & & & \\
\hline
\end{tabular}

Fuente: Registro Académico de la FACl, diciembre 2017

Tabla 3. Prueba de normalidad de Kolmogorov-Smirnov para una muestra

\begin{tabular}{|l|r|r|}
\hline & $\begin{array}{r}\text { PROMEDIO FINAL } \\
\text { ESTADÍSTICA } \\
\text { DESCRIPTIVA }\end{array}$ & $\begin{array}{c}\text { PROMEDIO FINAL } \\
\text { COMPUTACIÓN } \\
\text { MATEMÁTICA I }\end{array}$ \\
\hline $\mathrm{N}$ & 14 & 14 \\
Parámetros Media & 8,571 & 12,4286 \\
normales a,b Desviación típica & 3,3447 & 1,55486 \\
Diferencias más Absoluta &, 266 &, 143 \\
extremas Positiva $\quad$ Negativa &, 109 &, 109 \\
Z de Kolmogorov-Smirnov &,- 266 &,- 143 \\
Sig. asintót. (bilateral) &, 996 &, 536 \\
\hline
\end{tabular}

a. La distribución de contraste es la Normal.

b. Se han calculado a partir de los datos.

Fuente: Elaboración propia con software SPSS, agosto 2017

Tabla 4. Estadísticos descriptivos de la Asignatura Álgebra Moderna

\begin{tabular}{|l|r|r|r|r|c|}
\hline & N & Mínimo & Máximo & Media & Desv. típ. \\
\hline PROMEDIO FINAL & 14 & & & & \\
ALGEBRA MODERNA & 14 & 2,0 & 13,0 & 10,071 & 3,5834 \\
N válido (según lista) & 14 & & & & \\
\hline
\end{tabular}

Fuente: Registro Académico de la FACI, agosto 2017 
Tabla 5. Estadísticos descriptivos de la Asignatura Computación Matemática II

\begin{tabular}{|l|c|c|c|c|c|}
\hline & $\mathrm{N}$ & Mínimo & Máximo & Media & Desv. típ. \\
\hline PROMEDIO FINAL & 14 & 2,00 & 17,00 & 11,5714 & 4,60291 \\
COMPUTACIÓN & 14 & & & & \\
$\begin{array}{l}\text { MATEMÁTICA II } \\
\text { N válido (según lista) }\end{array}$ & 14 & & & \\
\hline
\end{tabular}

Fuente: Registro Académico de la FACl, agosto 2017

Tabla 6. Prueba de normalidad de Kolmogorov-Smirnov para una muestra

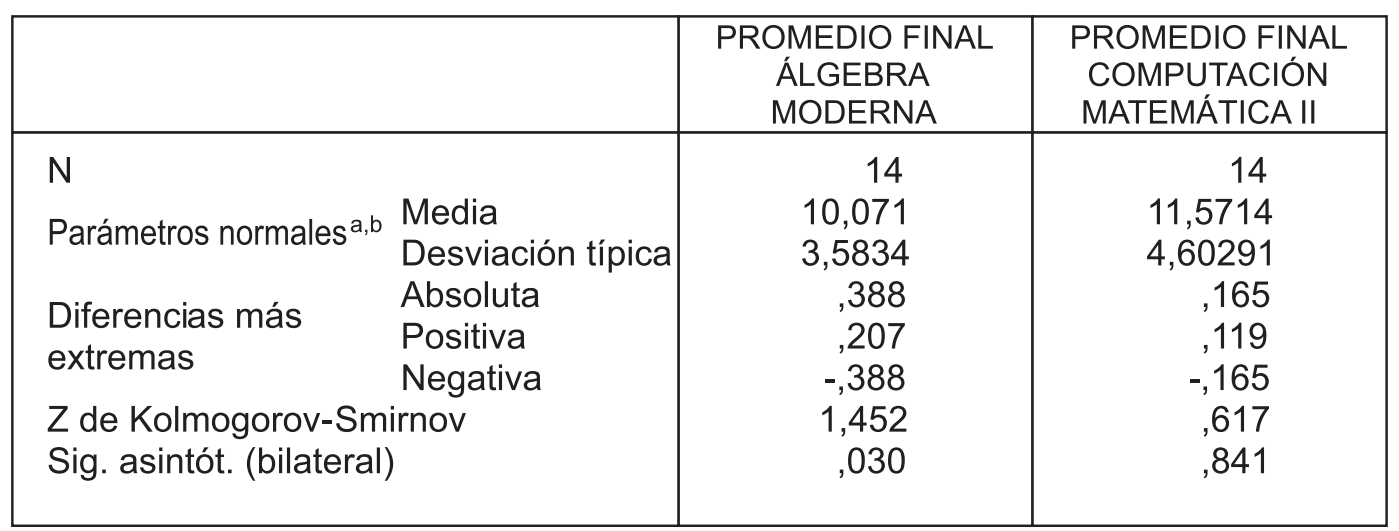

a. La distribución de contraste es la Normal.

b. Se han calculado a partir de los datos.

Fuente: Elaboración propia con software SPSS, agosto 2017

Tabla 7. Estadísticos descriptivos de la Asignatura Álgebra Lineal

\begin{tabular}{|l|c|c|c|c|c|}
\hline & N & Mínimo & Máximo & Media & Desv. típ. \\
\hline $\begin{array}{l}\text { PROMEDIO FINAL } \\
\text { ALGEBRA LINEAL } \\
\text { N válido (según lista) }\end{array}$ & 4 & 4,0 & 13,0 & 9,000 & 4,2426 \\
\hline
\end{tabular}

Tabla 8. Estadísticos descriptivos de la Asignatura Análisis Numérica I

\begin{tabular}{|l|l|l|l|l|l|}
\hline & N & Mínimo & Máximo & Media & Desv. típ. \\
\hline PROMEDIO FINAL & 4 & 11,00 & 18,00 & 13,2500 & 3,30404 \\
ANÁLISIS NUMÉRICO I & 4 & & & & \\
N válido (según lista) & 4 & & & & \\
\hline
\end{tabular}


Tabla 9. Prueba de normalidad de Kolmogorov-Smirnov para una muestra

\begin{tabular}{|ll|c|c|}
\hline & & $\begin{array}{c}\text { PROMEDIO FINAL } \\
\text { ALGEBRA LINEAL }\end{array}$ & $\begin{array}{c}\text { PROMEDIO FINAL } \\
\text { ANÁLISIS NUMÉRICO I }\end{array}$ \\
\hline $\mathrm{N}$ & Media & 4 & 4 \\
Parámetros normales & 9,000 & 13,2500 \\
& Desviación & 4,2426 & 3,30404 \\
& típica &, 260 &, 280 \\
Diferencias más extremas & Absoluta &, 181 &, 280 \\
& Positiva &,- 260 &,- 248 \\
Z de Kolmogorov-Smirnov &, 520 &, 560 \\
Sig. asintót. (bilateral) &, 949 &, 912 \\
\hline
\end{tabular}

a. La distribución de contraste es la Normal.

b. Se han calculado a partir de los datos.

Fuente: Elaboración propia con software SPSS, agosto 2017

Tabla 10. Estadísticos descriptivos de la Asignatura Análisis en Varias Variables

\begin{tabular}{|l|c|c|c|c|c|}
\hline & N & Mínimo & Máximo & Media & Desv. típ. \\
\hline PROMEDIO FINAL & & & & & \\
$\begin{array}{l}\text { ANÁLISIS EN VARIAS } \\
\text { VARIABLES } \\
\text { N válido (según lista) }\end{array}$ & 7 & 11,0 & 14,0 & 12,286 & 1,1127 \\
\hline
\end{tabular}

Fuente: Registro Académico de la FACl, agosto 2017

Tabla 11. Estadísticos descriptivos de la Asignatura Análisis Numérico II

\begin{tabular}{|l|c|c|c|c|c|}
\hline & N & Mínimo & Máximo & Media & Desv. típ. \\
\hline PROMEDIO FINAL & & & & & \\
ANÁLISIS NUMÉRICO II & 7 & 14,00 & 18,00 & 16,1429 & 1,77281 \\
N válido (según lista) & 7 & & & & \\
& & & & & \\
\hline
\end{tabular}

Tabla 12. Prueba de Kolmogorov-Smirnov para una muestra

\begin{tabular}{|ll|c|c|}
\hline & $\begin{array}{c}\text { PROMEDIO FINAL } \\
\text { ALGEBRA LINEAL }\end{array}$ & $\begin{array}{c}\text { PROMEDIO FINAL } \\
\text { ANÁLISIS NUMÉRICO I }\end{array}$ \\
\hline N & 7 & 7 \\
Parámetros $\quad$ Media & 12,286 & 16,1429 \\
normales & Desviación & 1,1127 & 1,77281 \\
& típica &, 173 &, 257 \\
Diferencias más & Absoluta &, 173 &, 172 \\
extremas $\quad$ Positiva & Negativa &,- 168 &,- 257 \\
Z de Kolmogorov-Smirnov &, 457 &, 680 \\
Sig. asintót. (bilateral) &, 985 &, 744 \\
\hline
\end{tabular}

a. La distribución de contraste es la Normal.

b. Se han calculado a partir de los datos.

Fuente: Elaboración propia con software SPSS, agosto 2017 
Tabla 13. Verificación de la hipótesis general para el II SEMESTRE Prueba de muestras relacionadas

\begin{tabular}{|c|c|c|c|c|c|c|c|c|c|}
\hline & \multicolumn{5}{|c|}{ Diferencias relacionadas } & & \multirow[t]{3}{*}{ Gl } & \multirow{3}{*}{$\begin{array}{c}\text { Sig. } \\
\text { (bilateral) }\end{array}$} \\
\hline & & \multirow[t]{2}{*}{ Media } & \multirow[t]{2}{*}{$\begin{array}{l}\text { Desviación } \\
\text { típ. }\end{array}$} & \multirow[t]{2}{*}{$\begin{array}{l}\text { Error } \\
\text { típ. de la } \\
\text { media }\end{array}$} & \multicolumn{2}{|c|}{$\begin{array}{c}95 \% \text { Intervalo de } \\
\text { confianza para la } \\
\text { diferencia }\end{array}$} & & & \\
\hline & & & & & Inferior & Superior & & & \\
\hline Par 1 & $\begin{array}{l}\text { PROMEDIO FINAL } \\
\text { COMPUTACIÓN } \\
\text { MATEMÁTICA I - } \\
\text { PROMEDIO FINAL } \\
\text { ESTADÍSTICA } \\
\text { DESCRIPTIVA }\end{array}$ & 3,85714 & 2,56776 & ,68626 & 2,37456 & 5,33973 & 5,620 & 13 &, 000 \\
\hline
\end{tabular}

Fuente: Elaboración propia con software SPSS, diciembre 2017

Tabla 14. Verificación de la hipótesis general para el III SEMESTRE Prueba de muestras relacionadas

\begin{tabular}{|c|c|c|c|c|c|c|c|c|c|}
\hline & \multicolumn{5}{|c|}{ Diferencias relacionadas } & & \multirow[t]{3}{*}{ Gl } & \multirow{3}{*}{$\begin{array}{c}\text { Sig. } \\
\text { (bilateral) }\end{array}$} \\
\hline & & \multirow[t]{2}{*}{ Media } & \multirow[t]{2}{*}{$\begin{array}{c}\text { Desviación } \\
\text { típ. }\end{array}$} & \multirow[t]{2}{*}{$\begin{array}{l}\text { Error } \\
\text { típ. de la } \\
\text { media }\end{array}$} & \multicolumn{2}{|c|}{$\begin{array}{c}95 \% \text { Intervalo de } \\
\text { confianza para la } \\
\text { diferencia }\end{array}$} & & & \\
\hline & & & & & Inferior & Superior & & & \\
\hline Par 1 & $\begin{array}{l}\text { PROMEDIO FINAL } \\
\text { COMPUTACIÓN } \\
\text { MATEMÁTICA I - } \\
\text { PROMEDIO FINAL } \\
\text { ESTADÍSTICA } \\
\text { DESCRIPTIVA }\end{array}$ & 3,85714 & 2,56776 & ,68626 & 2,37456 & 5,33973 & 5,620 & 13 & ,000 \\
\hline
\end{tabular}

Fuente: Elaboración propia con software SPSS, diciembre 2017

Tabla 15. Verificación de la hipótesis general para el V SEMESTRE Prueba de muestras relacionadas

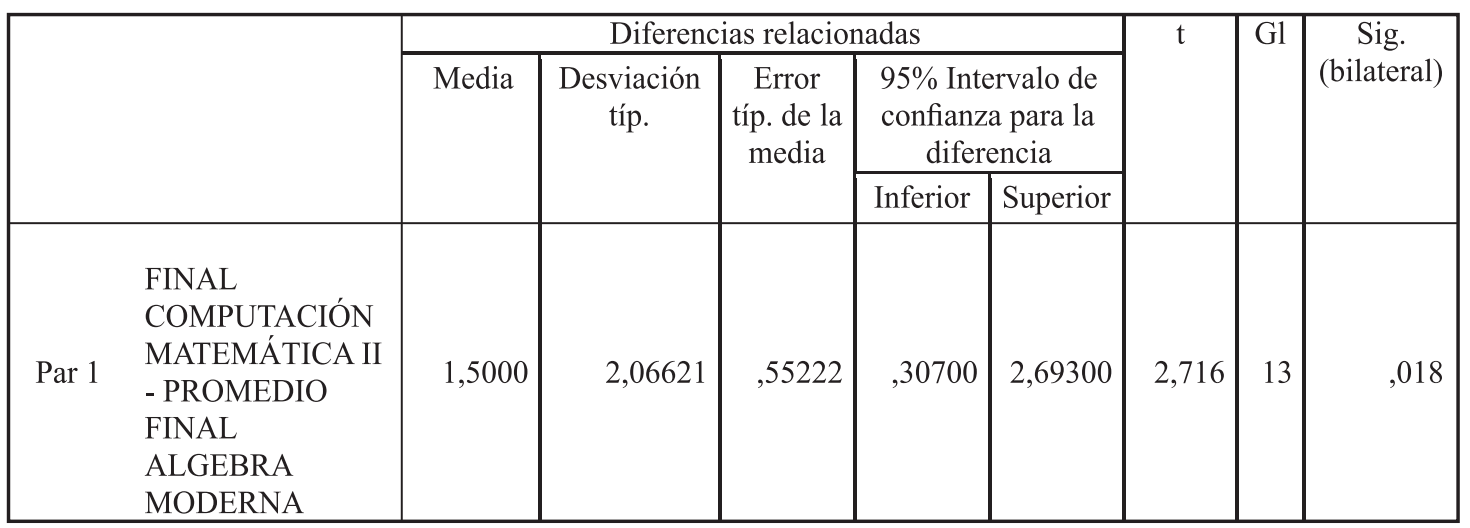

Fuente: Elaboración propia con software SPSS, diciembre 2017 
Tabla 16. Verificación de la hipótesis general para el VI SEMESTRE

Prueba de muestras relacionadas

\begin{tabular}{|c|c|c|c|c|c|c|c|c|c|}
\hline & & & Diferen & ias relacior & adas & & $\mathrm{t}$ & $\mathrm{gl}$ & Sig. \\
\hline & & Media & $\begin{array}{l}\text { Desviación } \\
\text { típ. }\end{array}$ & $\begin{array}{c}\text { Error típ. } \\
\text { de la } \\
\text { media }\end{array}$ & $\begin{array}{r}95 \% \text { Int } \\
\text { confian } \\
\text { dife }\end{array}$ & $\begin{array}{l}\text { rvalo de } \\
\text { a para la } \\
\text { encia }\end{array}$ & & & (bilateral) \\
\hline & & & & & Inferior & Superior & & & \\
\hline Par 1 & $\begin{array}{l}\text { PROMEDIO } \\
\text { FINAL } \\
\text { ANÁLISIS } \\
\text { NUMÉRICO II } \\
\text { - PROMEDIO } \\
\text { FINAL } \\
\text { ANÁLISIS EN } \\
\text { VARIAS } \\
\text { VARIABLES } \\
\end{array}$ & 3,85714 & _89974 & 34007 & 3,02503 & 4,68926 & 11,342 & 6 & ,000 \\
\hline
\end{tabular}

Fuente: Elaboración propia con software SPSS, diciembre 2017

\section{Verificación de las hipótesis}

\section{Hipótesis específicas}

a) La media de calificaciones en rendimiento académico del Grupo experimental, sin aplicar el Sistema de evaluación basado en competencias orientado hacia una Educación de calidad en estudiantes de la Escuela Profesional de Matemática de la Universidad Nacional Jorge Basadre Grohmann de Tacna - 2017 es baja. La cual se verifica al tener la media de 8,571 para la asignatura de ESTADÍSTICA DESCRIPTIVA (II semestre), la media de 10,071 para la asignatura de ÁLGEBRA MODERNA (III semestre), la media de 9,000 para la asignatura de ÁLGEBRA LINEAL (V semestre) y la media de 12,286 para la asignatura de ANÁLISIS EN VARIAS VARIABLE (VI semestre). Resultando una media en las cuatro asignaturas de 9,982 (Ver Cuadros 1, 4, 7 y 10).

b) La media de calificaciones en rendimiento académico del Grupo experimental, después de aplicar el Sistema de evaluación basado en competencias orientado hacia una Educación de calidad en estudiantes de la Escuela Profesional de Matemática de la Universidad Nacional Jorge Basadre Grohmann de Tacna - 2017 es alta. La cual se verifica al tener la media de 12,4286 para la asignatura de COMPUTACIÓN MATEMÁTICA I (II semestre), la media de 11,5714 para la asignatura de COMPUTACIÓN MATEMÁTICA II (III semestre), la media de 13,2500 para la asignatura de ÁNALISIS NUMÉRICO I (V semestre) y la media de 16,1429 para la asignatura de ANÁLISIS NUMÉRICO II (VI semestre). Resultando una media en las cuatro asignaturas de 13,348 (Ver Cuadros 2, $5,8$ y 11$)$

\section{Hipótesis general}

Existe una diferencia significativa entre la media de calificaciones en rendimiento académico de estudiantes del Grupo experimental, después de aplicar un Sistema de evaluación basado en competencias orientado hacia una Educación de calidad en estudiantes de la Escuela Profesional de Matemática de la Universidad Nacional Jorge Basadre Grohmann de Tacna - 2017.

\section{Verificación de la hipótesis general para el II SEMESTRE}

Interpretación: (Del Cuadro 13)

Debido a que el error típico de la diferencia es 0,000 entonces existe una diferencia significativa entre la media de calificaciones en rendimiento académico de estudiantes del Grupo experimental, después de aplicar un Sistema de evaluación basado en competencias orientado hacia una Educación de calidad en estudiantes de la Escuela Profesional de Matemática de la Universidad Nacional Jorge Basadre Grohmann de Tacna - 2017 correspondientes al II semestre (Asignaturas de ESTADÍSTICA DESCRIPTIVA y COMPUTACIÓN MATEMÁTICAI). 


\section{Verificación de la hipótesis general para el III SEMESTRE}

Interpretación: (Del Cuadro 14)

Debido a que el error típico de la diferencia es 0,018 entonces existe una diferencia significativa entre la media de calificaciones en rendimiento académico de estudiantes del Grupo experimental, después de aplicar un Sistema de evaluación basado en competencias orientado hacia una Educación de calidad en estudiantes de la Escuela Profesional de Matemática de la Universidad Nacional Jorge Basadre Grohmann de Tacna - 2017 correspondientes al III semestre (Asignaturas de ÁLGEBRA MODERNA y COMPUTACIÓN MATEMÁTICAII).

\section{Verificación de la hipótesis general para el V SEMESTRE}

Interpretación: (Del Cuadro 15)

Debido a que el p-valor es 0,042 entonces existe una diferencia significativa entre la media de calificaciones en rendimiento académico de estudiantes del Grupo experimental, después de aplicar un Sistema de evaluación basado en competencias orientado hacia una Educación de calidad en estudiantes de la Escuela Profesional de Matemática de la Universidad Nacional Jorge Basadre Grohmann de Tacna - 2017 correspondientes al V SEMESTRE (Asignaturas de ÁLGEBRA LINEAL y ANÁLISIS NUMÉRICO I).

\section{Verificación de la hipótesis general para el VI SEMESTRE}

Interpretación: (Del Cuadro 16)

Debido a que el p-valor es 0,000 entonces existe una diferencia significativa entre la media de calificaciones en rendimiento académico de estudiantes del Grupo experimental, después de aplicar un Sistema de evaluación basado en competencias orientado hacia una Educación de calidad en estudiantes de la Escuela Profesional de Matemática de la Universidad Nacional Jorge Basadre Grohmann de Tacna - 2017 correspondientes al VI SEMESTRE (Asignaturas de ANÁLISIS EN VARIAS VARIABLES Y ANÁLISIS NUMÉRICO II).

\section{DISCUSIÓN}

Respecto a las medias de calificaciones la media en rendimiento académico SIN aplicar el sistema de evaluación basado en competencias, después de analizar descriptivamente por una media de 8,571 en la Asignatura de ESTADÍSTICA DESCRIPTIVA (II Semestre), una media de 11,571 en la Asignatura de ÁLGEBRA MODERNA (III Semestre), una media de 9,000 en la Asignatura de ÁLGEBRA LINEAL (V Semestre) y una media de 12,286 en la Asignatura de ANÁLISIS EN VARIAS VARIABLES (VI Semestre). Resultando una media en las cuatro asignaturas de 9,982, y la media de calificaciones en rendimiento académico APLICANDO el sistema de evaluación basado en competencias, después de analizar descriptivamente por una media de 12,4286 en la Asignatura de COMPUTACIÓN MATEMÁTICA I (II Semestre), una media de 11,5714 en la Asignatura de COMPUTACIÓN MATEMÁTICA II (III Semestre), una media de 13,2500 en la Asignatura de ANÁLISIS NUMÉRICO I (V Semestre) y una media de 16,1429 en la Asignatura de ANÁLISIS NUMÉRICO II (VI Semestre). Resultando una media en las cuatro asignaturas de 13,348 , concordamos con la investigación de Vargas \& Otros (2016), cuando llega a la conclusión siguiente: En alumnos ingresantes de la Escuela Profesional de Matemática de la Universidad Nacional Jorge Basadre Grohmann de Tacna - 2016, la media de calificaciones en rendimiento académico SIN aplicar el sistema de evaluación basado en competencias es baja. Detectado, después de analizar descriptivamente por una media de 8,706 en la Asignatura de Geometría Analítica (I Ciclo) y una media de 9,098 en la Asignatura de Estadística Descriptiva (II Ciclo).

Por otro lado, la media de calificaciones en rendimiento académico APLICANDO el sistema de evaluación basado en competencias después de analizar Por otro lado, la media de calificaciones en rendimiento académico APLICANDO el sistema de evaluación basado en competencias después de analizar descriptivamente por una media del12,4286 en la Asignatura de COMPUTACIÓN MATEMATICA II (III Semestre), una media de 13,2500 en la Asignatura de ANÁLISIS NUMERICO I (V Semestre) y una media de 16,1429 en la Asignatura de ANÁLISIS 
NUMERICO II (VI Semestre), resultando una media en las cuatro asignaturas de 13,348 igualmente, concordamos con la investigación de Vargas \& Otros (2016), cuando llega a la conclusión siguiente: En alumnos ingresantes de la Escuela Profesional de Matemática de la Universidad Nacional Jorge Basadre Grohmann de Tacna - 2016, la media de calificaciones en rendimiento académico DESPUES de aplicar el sistema de evaluación basada en competencias es alta. Detectado, después de analizar descriptivamente por una media de 13,556 en la Asignatura de Complemento de Matemáticas (I Ciclo) y una media de 13,609 en la Asignatura de media de 13,609 en la Asignatura de Computación Matemática I (II Ciclo).

\section{CONCLUSIONES}

En estudiantes de la Escuela Profesional de Matemática de la Universidad Nacional Jorge Basadre Grohmann de Tacna - 2017, la media de calificaciones en rendimiento académico SIN aplicar el sistema de evaluación basado en competencias es baja. Detectado, después de analizar descriptivamente por una media de 8,571 en Ia Asignatura de ESTADÍSTICA DESCRIPTIVA (II Semestre), por una media de 11,571 en la Asignatura de ÁLGEBRA MODERNA (III Semestre), una media de 9,000 en la Asignatura de ÁLGEBRA LINEAL ( $V$ Semestre) y una media de 12,286 en la Asignatura de ANÁLISIS EN VARIAS VARIABLES (VI Semestre). Resultando una media en las cuatro asignaturas de 9,982.

En estudiantes de la Escuela Profesional de Matemática de la Universidad Nacional Jorge Basadre Grohmann de Tacna - 2017, la media de calificaciones en rendimiento académico APLICANDO el sistema de evaluación basado en competencias es alta. Detectado, después de analizar descriptivamente por una media de 12,4286 en la Asignatura de COMPUTACIÓN MATEMÁTICA I (II Semestre), una media de 11,5714 en la Asignatura de COMPUTACIÓN MATEMÁTICA II (III Semestre), una media de 13,2500 en la Asignatura de ANÁLISIS NUMÉRICO I (V Semestre) y una media de
16,1429 en la Asignatura de ANÁLISIS NUMÉRICO II (VI Semestre). Resultando una media en las cuatro asignaturas de 13,348 .

Existe una diferencia significativa entre la media de calificaciones en rendimiento académico, sin aplicar y después de aplicar el sistema de evaluación baso en competencias en estudiantes de la Escuela Profesional de Matemática de la Universidad Nacional Jorge Basadre Grohmann de Tacna - 2017. Detectado, después de aplicar la prueba T para diferencia de medias, por un P-Valor $=$ $\mathbf{0 , 0 0 0}$ en el II Semestre, por un P-Valor $=$ 0,018 en el III Semestre, un P-Valor $=\mathbf{0 , 0 4 2}$ en el V Semestre y un $\mathbf{P}$-Valor $\mathbf{0} \mathbf{0 , 0 0 0}$ en el VI Semestre los cuales son menores que 0,05.

\section{REFERENCIAS BIBLIOGRÁFICAS}

Carrillo, G. (2015). "El currículo por competencias en la Educación Superior". Lima: Nova Print S.A.C. Recuperado de: http://vicerrectorado.pucp.edu.pe/academic o/wp-content/uploads/2015/03/curriculo _competencias.pdf

[Consulta 04 de enero del 2017]

Lorenzana R. (2012). La evaluación de los aprendizajes basada en competencias en la enseñanza universitaria. (Tesis de doctoral). Universität Flensburg, Honduras, Centroamérica.

Mendoza J. \& Otros (2014). Diseño curricular de la Escuela Académica Profesional de Matemática. Universidad Nacional Jorge Basadre Grohmann, Tacna, Perú. (Resolución Rectoral No 3363-2014UN/JBG)

Vargas H.; Solórzano E. \& Huamán I. (2017). Sistema de evaluación basado en competencias orientado hacia una Educación de calidad y su influencia en el rendimiento académico en alumnos ingresantes de la Escuela Profesional de Matemática de la Universidad Nacional Jorge Basadre Grohmann de Tacna - 2016. Revista de Investigación de la Facultad de Ciencias, Universidad Nacional Jorge Basadre Grohmann. Tacna-PERÚ. 1(1), 43-49. 